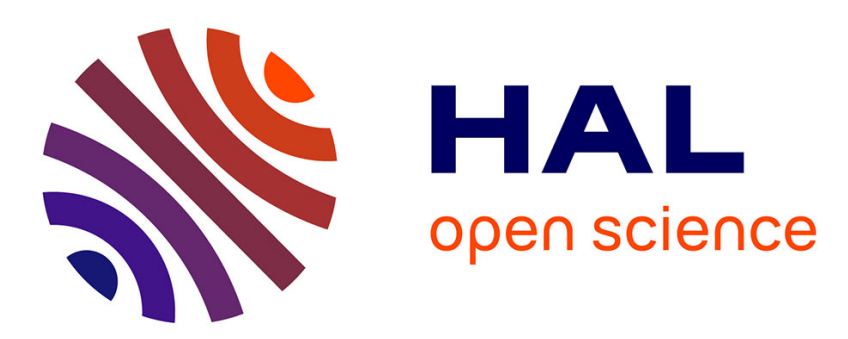

\title{
Immunotoxicity of poly (lactic-co-glycolic acid) nanoparticles: influence of surface properties on dendritic cell activation
}

S. Barillet, E. Fattal, S. Mura, N. Tsapis, M. Pallardy, H. Hillaireau, S. Kerdine-Römer

\section{To cite this version:}

S. Barillet, E. Fattal, S. Mura, N. Tsapis, M. Pallardy, et al.. Immunotoxicity of poly (lactic-co-glycolic acid) nanoparticles: influence of surface properties on dendritic cell activation. Nanotoxicology, 2019, 13 (5), pp.606-622. 10.1080/17435390.2018.1564078 . hal-02323703

\section{HAL Id: hal-02323703 \\ https://hal.science/hal-02323703}

Submitted on 7 Nov 2019

HAL is a multi-disciplinary open access archive for the deposit and dissemination of scientific research documents, whether they are published or not. The documents may come from teaching and research institutions in France or abroad, or from public or private research centers.
L'archive ouverte pluridisciplinaire HAL, est destinée au dépôt et à la diffusion de documents scientifiques de niveau recherche, publiés ou non, émanant des établissements d'enseignement et de recherche français ou étrangers, des laboratoires publics ou privés. 


\section{Immunotoxicity of poly(lactic-co-glycolic acid) nanoparticles: influence 2 of surface properties on dendritic cell activation}

3 Barillet S. ${ }^{a}$, Fattal E. ${ }^{\text {b }}$, Mura S. ${ }^{\text {b }}$, Tsapis N. ${ }^{b}$, Pallardy M. ${ }^{a}$, Hillaireau H. ${ }^{\text {,o }}$, 4 Kerdine-Römer S., ${ }^{\mathrm{a}, *}$

5 a UMR996 Inflammation, Chemokines and Immunopathology, INSERM, Univ. Paris6 Sud, Université Paris-Saclay, Châtenay-Malabry, France, ${ }^{b}$ Institut Galien Paris-Sud, 7 Univ. Paris-Sud, CNRS, Université Paris-Saclay, Châtenay Malabry, France.

8 equal contribution

9 *corresponding author: UMR996, Faculté de Pharmacie, Univ. Paris-Sud, 5 rue J.B. 10 Clément, 92296 Châtenay-Malabry, France.

11 email: $\underline{\text { saadia.kerdine-romer@u-psud.fr }}$

13 Running Title

14 Immunotoxicity of PLGA nanoparticles on dendritic cell activation 15 16 
2 Immunotoxicity of poly(lactic-co-glycolic acid) nanoparticles: influence 3 of surface properties on dendritic cell activation

4 Modified nanoparticles (NPs) can interact with the immune system by causing its 5 activation to fight tumors or for vaccination. During this activation, dendritic cells (DCs) 6 are effective in generating robust immune response. However, the effect of nanomaterials 7 on dendritic cell (DC) maturation, and the associated adjuvant effect, should be assessed 8 as a novel biocompatibility criteria for biomaterials since immune consequences may 9 constitute potential complications in nanomedicine. Among emerging biomaterials, 10 poly(lactic-co-glycolic acid) NPs (PLGA NPs) are widely explored for various 11 applications in which the degree of desired adjuvant effect may vary. As contradictory 12 results are reported regarding their effects on DCs, we aimed at clarifying this point with 13 particular emphasis on the relative impact of particle surface properties. To that end, NP 14 uptake and effects on the viability, phenotype and secretory activity of DC primary 15 cultures. Intracellular signaling pathways were additionally explored were evaluated. 16 Immature human and murine DCs, were exposed to cationic, neutral, or anionic PLGA 17 NPs. Particle uptake was first assessed by both confocal microscopy and flow cytometry. 18 Cell viability was then evaluated prior to the study of maturation by examination of both 19 surface marker expression and cytokine release. Our results demonstrate that PLGA NPs 20 are rapidly engulfed by DCs and do not exert cytotoxic effects. However, upon exposure 21 to PLGA NPs, DCs showed phenotypes and cytokine secretion profiles consistent with 22 maturation which resulted, at least in part, from the transient intracellular activation of 23 mitogen-activated protein kinases (MAPKs). Interestingly, NP-specific stimulation 24 patterns were observed since NP surface properties had sensible influence on the various 25 parameters measured. 


\section{Introduction}

2 Nanotechnology applications in medicine, termed as nanomedicine, have opened doors

3 to a plethora of possible answers to several impediments of conventional medicine.

4 Among the broad panel of available materials in nanomedicine, poly(lactic-co-glycolic)

5 acid (PLGA) has been extensively investigated, as this copolymer is generally viewed

6 as biocompatible and biodegradable (Panyam and Labhasetwar, 2003 ; Bala et al., 2004;

7 Mundargi et al., 2008; Lu et al., 2009; Xu et al., 2009). It has been approved by the US

8 FDA for drug delivery and other applications in clinics, and widely investigated for

9 applications in cardiovascular diseases, cancer, vaccine and tissue engineering ( $\mathrm{Lu}$ et

10 al., 2009). In this context, NPs of PLGA are explored as carriers for the controlled

11 delivery of small molecules such as anticancer cytotoxic compounds, and

12 macromolecular therapeutics such as proteins, peptides, vaccines, genes, antigens and

13 growth factors (Mundargi et al., 2008). Because of this increasing interest for these

14 PLGA NPs, it is obvious that a careful assessment of their potential toxicity is crucial.

15 Despite such a need for risk assessment studies to be carried out, the current state of

16 knowledge about biological effects and toxicity of PLGA NPs is well characterized for

17 some aspects (Bisht \& Rupenthal, 2016) but remains limited for most of exposure

18 routes.

19 In the specific context of nanotoxicology, the field of immunotoxicology is

20 gaining a growing interest, as the interactions between NPs and the immune system

21 have proven pivotal in the response to NP exposure (Dobrovolskaia \& McNeil, 2007;

22 Dobrovolskaia et al., 2009; Zolnik et al., 2010; Blank et al., 2011; Di Gioacchino et al.,

23 2011; Oostingh et al., 2011). The immune response to foreign chemical and/or

24 biological molecules as well as to particles is typically initiated through a protective

25 innate inflammatory response. With the exception of some scarce studies, PLGA-based 
1 NPs would not seem to induce such an innate response, as shown by our group and

2 others (Dailey et al., 2006; Mura et al., 2011a, 2011b, Aragao-Santiago et al., 2015). In

3 particular, PLGA-based NPs induced moderate but significant suppression of of T-

4 lymphocytes proliferation (Aragao-Santiago et al., 2015). However, little is known

5 about the effects of PLGA NPs on the activation of antigen-specific cells (APCs) such

6 as dendritic cells (DCs). DCs play a major role in innate immunity by driving the

7 specific reaction by the adaptive immune system. A myriad of cytokines (TNF- $\alpha$,

8 GMCSF, IL-1, ...) may be produced by our innate immune system in response to a

9 xenobiotic allowing inflammation and contributing to immunotoxic effects (Petrarca et 10 al., 2015).

11 Given the importance of DCs, NP impact on these cells raises growing concerns.

12 Among the deleterious effects of NPs on DCs currently expected, immunosuppression

13 has been suggested (Ilinskaya \& Dobrovolskaia, 2014). More insidiously, NPs could act

14 as immune adjuvants, inducing exacerbation or modification of the type of immune

15 response to a given antigen, hence resulting in hypersensitivity or allergic reactions.

16 Possible impacts on the immune system stress the need for a careful

17 immunotoxicological evaluation, particularly for regulatory purposes (Dobrovolskaia et 18 al. 2009, 2016).

19 To elucidate PLGA NP-dependent outcomes on DCs, we herein present an in 20 vitro study of the effect of 100-250 nm PLGA NPs on both human monocyte-derived 21 DCs (MoDCs) and murine bone-marrow derived DCs (BMDCs). Since the performance 22 of nanotechnology-based drug delivery systems is strongly influenced mostly by surface 23 properties (charge and hydrophilicity), surfactants may have a strong impact on 24 resulting biological effects of particles, especially in terms of uptake and intracellular 25 fate (Bhardwaj et al., 2005; Yue et al., 2011; Sadat et al., 2016; Chen et al., 2018). We 
1 therefore conducted our study on PLGA NPs formulated with three different stabilizers:

2 polyvinyl alcohol (PVA), chitosan (CS) or poloxamer 188 (commercially named

3 Pluronic F68 [PF68]), resulting in neutral, positively or negatively charged particles,

4 respectively. NP uptake by DCs was first examined. Viability, phenotypical changes

5 and cytokine release of DCs were then assessed. To further understand underlying

6 mechanisms involved in observed responses, intracellular signaling pathways were also

7 investigated. We believe that this approach assessing the immune response to PLGA

8 NPs may contribute to a better understanding of their biological fate and to a robust

9 assessment for their use as pharmaceutical ingredients.

\section{Methods}

\section{Preparation of nanoparticle suspensions}

12 PLGA NPs were prepared by solvent evaporation-based methods (Mura et al., 2011a).

13 Positively and negatively charged as well as neutral NPs were tailored by coating their

14 surface with chitosan (CS, Protasan® UP CL113, 75\%-90\% deacetylation, molecular weight 50-150 kD), poloxamer 188 (commercially named Pluronic F68 [PF68]), or

16 polyvinyl alcohol (PVA, 87\%-89\% hydrolyzed, molecular weight 30-70 kDa), 17 respectively.

Neutral NPs (PLGA/PVA NPs) were prepared by dissolving $100 \mathrm{mg}$ of PLGA

19 (75:25 Resomer RG756) in $5 \mathrm{~mL}$ of a dichloromethane/acetone (1/1 v/v) mixture. This organic solution was pre-emulsified with $20 \mathrm{~mL}$ of a $0.25 \%(\mathrm{w} / \mathrm{v})$ PVA (molecular

21 weight $30-70 \mathrm{kDa}$ ) aqueous solution by vortexing for one minute. The pre-emulsion

22 was kept on ice and sonicated for one minute using a VibraCell sonicator (Fisher

23 Scientific, Illkirch, France) at $40 \%$ power. As for positively charged NPs (PLGA/CS

24 NPs), the organic solution of PLGA was pre-emulsified with a $0.6 \%(\mathrm{w} / \mathrm{v})$ aqueous CS 
1 solution containing $0.5 \%(\mathrm{w} / \mathrm{v})$ of Mowiol 4-88 PVA by vortexing for one minute. This

2 preemulsion was kept on ice and sonicated for 2 minutes using a VibraCell sonicator at

$340 \%$ power. Then, for both the neutral and positively charged NPs, the organic phase

4 was allowed to evaporate at room temperature with magnetic stirring $(600 \mathrm{rpm})$. NP

5 dispersion was then completed to $20 \mathrm{~mL}$. The excess of stabilizers was removed by

6 centrifugation of NPs at $37,000 \times g$ for one hour at $4{ }^{\circ} \mathrm{C}$, and the pellet of NPs was

7 resuspended in ultrapure water.

8 Negatively charged NPs (PLGA/PF68 NPs) were prepared using PF68 as

9 stabilizer. PLGA was dissolved in $10 \mathrm{~mL}$ ethyl acetate and added dropwise into $20 \mathrm{~mL}$

10 of a $1 \%(\mathrm{w} / \mathrm{v})$ PF68 aqueous solution under vigorous magnetic stirring. The pre-

11 emulsion was vortexed for one minute and then sonicated for another minute using a

12 VibraCell sonicator at $40 \%$ power. Water $(20 \mathrm{~mL})$ was added to the emulsion, in order

13 to promote diffusion of the organic solvent into the external phase, leading to formation

14 of the NPs. The organic phase was allowed to evaporate at room temperature with

15 magnetic stirring $(600 \mathrm{rpm})$.

16 Rhodamine B-labelled NPs were prepared as described earlier (Mura et al., 17 2011a) by dissolving in the organic solvent a 70/30\% (w/w) mixture of PLGA 18 (Resomer RG756) and PLGA covalently bound to rhodamine B.

19 Nanoparticle suspension characterization

20 NPs were characterized in terms of size, zeta potential and surface chemical 21 composition, confirming modifications provided by hydrophilic stabilizers. NP size 22 (hydrodynamic diameter) and polydispersity were determined after dilution in MilliQ® 23 water at $25^{\circ} \mathrm{C}$ by dynamic light scattering using a Nano ZS (Malvern Instrument, UK) 24 set at a $173^{\circ}$ scattering angle. The zeta potential was determined using the same 25 instrument after dilution in $1 \mathrm{mM} \mathrm{NaCl}$. All measurements were done in triplicate. 
The amount of stabilizers associated to NPs after their purification was assessed

2 through the formation of colored complexes of PVA and CS, or by $1 \mathrm{H}$ NMR in the case

3 of PF68, as previously described (Mura et al., 2011a).

The morphology of NPs in aqueous suspension was studied by transmission

5 electron microscopy (TEM) using a JEOL JEM-1400 microscope operating at $80 \mathrm{kV}$

6 (filament current of $55 \mu \mathrm{A}$ ). $5 \mu \mathrm{L}$ of $\mathrm{NP}$ aqueous suspensions was deposited onto

7 copper grids covered with formvar film (400 mesh) for one minute. PLGA samples

8 were then stained using 2\% phosphotungstic acid (PLGA/PVA, PLGA/PF68) or 2\%

9 uranyl acetate (PLGA/CS) for 30 seconds. The excess liquid was blotted off using filter

10 paper, and the grids were dried before observation. Images were acquired using a

11 postcolumn high-resolution (11 megapixels) high-speed camera (SC1000 Orius; Gatan)

12 and processed with Digital Micrograph (Gatan) and ImageJ.

13 Endotoxin level in NP suspensions was $<0.05 \mathrm{EU} / \mathrm{ml}$ as tested by limulus

14 amebocyte lysat (LAL) assay (GenScript, Piscataway, NJ, USA).

\section{Generation of primary cultures of human and murine dendritic cells}

16 The generation of human DCs derived from monocytes (MoDCs) was performed as

17 described elsewhere (Antonios et al., 2010) from human peripheral blood supplied by

18 the French Blood Institute (EFS, Rungis, France). First, peripheral blood mononuclear

19 cells (PBMCs) were sorted from phosphate buffer saline (PBS) diluted buffy coats by

20 density centrifugation on a Ficoll gradient $\left(650 \times g, 20 \mathrm{~min}, 19^{\circ} \mathrm{C}\right)$. Monocytes were

21 then isolated through positive magnetic selection using MiniMacs separation columns

22 and anti-CD14 ${ }^{+}$antibodies coated on magnetic beads (Miltenyi Biotec, Paris, France).

23 Finally, CD14 ${ }^{+}$cells were differentiated immature monocyte-derived DCs (MoDCs) for

245 days in RPMI 1640 supplemented with Glutamax-I, $10 \%$ heat inactivated fetal calf

25 serum (FCS), $550 \mathrm{U} / \mathrm{mL}$ granulocyte-macrophage colony-stimulating factor (rh-GM- 
$1 \mathrm{CSF}$ ), $550 \mathrm{U} / \mathrm{mL}$ interleukin-4 (rh-IL4), $1 \%$ sodium pyruvate, streptomycin and 2 penicillin.

The generation of murine DCs (BM-DCs) was performed as described

4 elsewhere (El Ali et al., 2017). In brief, DCs were derived from bone-marrow

5 hematopoietic progenitors collected from the femurs and tibiae of 8 -10-week-old

6 C57BL/6 mice (Janvier SAS, Saint Berthevin, France). Cells were then differentiated to

7 immature BMDCs for 7 days in Iscove's modified Dulbecco's medium (IMDM)

8 containing $10 \% \mathrm{FCS}, 50 \mu \mathrm{M} \beta$-mercaptoethanol and $10 \%$ culture supernatant from

9 mouse GM-CSF-producing J558 cells (a kind gift of S. Amigorena, Institut Curie),

10 coresponding to a final concentration of about $40 \mathrm{ng} / \mathrm{mL}$ GM-CSF. Mice were handled

11 in accordance with the principles and procedures in accordance with the decree $\mathrm{n}^{\circ} 2013$ -

121118 of 1 February 2013 on the protection of animals, used for scientific purposes

13 Article R214.89.

15 In vitro exposure of immature dendritic cells to PLGA nanoparticles

16 After proper differentiation, immature DCs were collected, washed, and resuspended in 17 fresh complete culture medium (RPMI or IMDM for human and murine cells 18 respectively, supplemented with $10 \%$ FCS, $1 \%$ sodium pyruvate and antibiotics) at a

19 final density of $10^{6}$ cells/ml. Cells were then exposed to either PLGA NPs, their vehicle 20 (distilled water, negative control), or any other chemical or biological molecule that 21 would constitute an adequate positive control for a given analysis.

\section{Nanoparticle internalization by DCs}

23 For microscopy analysis, after exposure to NPs, cells were harvested, washed with cold 24 PBS, fixed for $15 \mathrm{~min}$ in PBS supplemented with $2 \%$ paraformaldehyde (PFA) and $251.5 \%$ sucrose. Cells were then washed twice with PBS, incubated for $10 \mathrm{~min}$ in $50 \mathrm{mM}$ 
1 ammonium chloride, and washed twice with PBS once again. Cells were then mounted

2 onto microscope slides using an appropriate mounting medium (DakoCytomation

3 mounting medium supplemented with 4',6-diamidino-2-phenylindole (DAPI) for

4 nucleus labeling) and examined under a confocal microscope equipped with

5 epifluorescence.

6 For cytometry analysis, after exposure to rhodamine-labelled NPs, cells were

7 harvested, washed with cold PBS, and analyzed on a FACSCalibur@ cell analyzer using

8 the CellQuest ${ }^{\circledR}$ software (BD Biosciences). Results were expressed as percentage of

9 positive cells.

\section{Cytotoxicity evaluation}

11 Cell viability was first assessed by the MTT (methylthiazolyldiphenyl-tetrazolium

12 bromide) test. Immature cells were seeded at $10^{5}$ cells $/ 100 \mu \mathrm{L} /$ well into 96 -well plates

13 and exposed to $1-400 \mu \mathrm{g} / \mathrm{mL}$ PLGA NPs for $24 \mathrm{~h}$ (5 replicate wells per treatment

14 group). At the end of the exposure, $10 \mu \mathrm{L}$ of a $5 \mathrm{mg} / \mathrm{ml}$ MTT solution was added to

15 each well. After $3 \mathrm{~h}$ at $37^{\circ} \mathrm{C}$, medium was replaced by $100 \mu \mathrm{L}$ of dimethyl sulfoxide

16 (DMSO) to dissolve the resulting formazan crystals and optical density was measured at

$17550 \mathrm{~nm}$ with the reference filter set to $620 \mathrm{~nm}$. Cell viability values were finally

18 determined as percent differences relative to untreated (negative) control values.

19 The trypan blue dye exclusion method was additionally used to assess

20 cytotoxicity. Viable and dead cells were counted with a hemocytometer, distincted

21 thanks to the use of trypan blue, a vital stain that only enters dead cells.

22 Obtained cytotoxicity curves were used to identify NP exposure conditions that

23 do not induce more than $30 \%$ cell death so as to investigate NP effects on DC 24 maturation. 


\section{Phenotypic analysis}

2 To assess the phenotypic effects of NP exposure on DCs, several surface maturation

3 markers were studied. After $24 \mathrm{~h}$ of exposure to either $200 \mu \mathrm{g} / \mathrm{mL}$ PLGA NPs, distilled

4 water (negative control) or $25 \mathrm{ng} / \mathrm{mL}$ lipopolysaccharide (LPS, positive control), DCs

5 were collected, washed with PBS and incubated for $15 \mathrm{~min}$ in Fc Block (BD

6 Biosciences, Le Pont de Claix, France) to avoid antibody preparations binding via their

7 Fc portions to low-affinity receptors for complexed IgG. Cells were then incubated for

820 min either with fluorochrome-conjugated monoclonal antibodies (mAbs) or with

9 appropriate isotype control antibodies (IgGs). For MoDCs, the following mouse anti-

10 human mAbs were used: FITC-conjugated anti-CD40 (clone 5C3), APC-conjuguated

11 anti-CD54 (ICAM-1,clone HA58), FITC-conjuguated anti-CD80 (clone L307.4), APC-

12 conjugated anti-CD83 (clone HB15e), FITC-conjugated anti-CD86 (clone 2331(FUN-

13 1)), FITC-conjugated anti-CD274 (PD-L1, clone MIH1), PE-conjugated anti-CD197

14 (CCR7) and FITC-conjugated anti-HLA-DR (clone G46-6) (BD Biosciences). For

15 BMDCs, the following rat anti-mouse mAbs were used: FITC-conjugated anti-CD40

16 (clone 3/23), FITC-conjugated anti-CD86 (clone GL1), PE-conjugated anti-CD197

17 (CCR7, clone 4B12) (Beckman Coulter - Immunotech, Marseille, France). After mAbs

18 or IgGs incubation, DCs were washed twice with PBS supplemented with $0.5 \%$ BSA,

19 then once with PBS, and finally fixed in PBS supplemented with $1 \%$ PFA.

20 Cells unstimuled with NP were used to determine nonspecific staining. A minimum of 2120,000 cells were analyzed on a FACSCalibur cell analyzer with CellQuest software 22 (BD Biosciences). Living cells were identified on the basis of their physical 23 characteristics [forward scatter and side scatter. Results were expressed as percentage of 24 positive cells (i.e., cells that express the marker) as well as using the relative 25 fluorescence intensity (RFI, i.e. mean fluorescence intensity of cells incubated with a 
1 given $\mathrm{mAb}$ divided by mean fluorescence intensity of cells incubated with its

2 corresponding isotype) and the "fold increase RFI" (i.e. NP- or LPS-exposed cells RFI

3 divided by control cells RFI). This latter parameter (fold increase RFI) therefore reflects

4 the mean expression level of a given maturation marker within the total cell population.

\section{Cytokine analysis}

6 The BD Biosciences cytometric bead array (CBA) technique was performed to measure

7 cytokine concentrations within DC supernatants at the end of NP exposure. This

8 technique employs microbeads coated with a capture antibody specific for a given

9 soluble protein. For human MoDC supernatants, tumor necrosis factor (TNF)- $\alpha$, 10 interleukin (IL)-1 $\beta$, IL-6, IL-8, IL-10, interferon- $\gamma$-induced protein (IP)-10 (also known

11 as CXCL10), monocyte chemotactic protein (MCP)-1 (also known as CCL2) and

12 RANTES (regulated upon activation, normal T-cell expressed and secreted, also known

13 as CCL5) were tested. For murine BMDC supernatants, TNF- $\alpha$, IL-6, IL-10, MCP-1

14 and CCL5 were tested.

15 Briefly, cell culture supernatants were either diluted or not and serial dilutions of

16 each cytokine standard (from 10 to $2500 \mathrm{pg} / \mathrm{mL}$ ) were prepared. Test samples and

17 standard dilutions ( $50 \mu \mathrm{L} /$ test $)$ were then incubated with capture beads for $1 \mathrm{~h}$ at room

18 temperature, protected from light. Then, PE detection reagents were added and an

19 additional incubation step was performed ( $2 \mathrm{~h}$, room temperature, protected from light).

20 After proper washes, a three-color cytometric analysis was carried out using a

21 FACScalibur flow cytometer (BD Biosciences). Results were generated using the FCAP 22 Array $^{\mathrm{TM}}$ Software. 


\section{$1 \quad$ Intracellular signalling pathways}

2 Western blot analyses of both mitogen activated protein kinases (MAPK) and NFא-B

3 (nuclear factor kappa-light-chain-enhancer of activated B cells) were performed as

4 previously described (Larangé et al., 2012). Immature DCs $\left(10^{6}\right.$ cells $\left./ \mathrm{mL}\right)$ were either

5 exposed to distilled water, PLGA NPs $(200 \mu \mathrm{g} / \mathrm{mL})$, or LPS (25 ng/mL) for $15,30,60$

6 or $120 \mathrm{~min}$. At the end of their stimulation, cells were harvested, washed with PBS and

7 pelleted by centrifugation. Cell lysates were then prepared by resuspending cell pellets

8 in lysis buffer (20 mM Tris pH 7.4, $137 \mathrm{mM} \mathrm{NaCl,} 2$ mM EDTA pH 7.4, 2 mM sodium

9 pyrophosphate, $1 \%$ Triton X-100, $10 \%$ glycerol, $1 \mathrm{mM}$ PMSF, $1 \mathrm{mM}$ sodium

10 orthovanadate, $25 \mathrm{mM} \beta$-glycerophophate, $10 \mu \mathrm{g} / \mathrm{mL}$ aprotinin, $10 \mu \mathrm{g} / \mathrm{mL}$ leupeptin and

$11100 \mu \mathrm{g} / \mathrm{mL}$ pepstatin). Total protein extracts were then collected after a $20 \mathrm{~min}$

$1215,000 \mathrm{rpm}$ centrifugation. Fifty $\mu \mathrm{g}$ of denaturated protein were then loaded onto

$1312.5 \%$ SDS-PAGE gel and transferred on polyvinylidene fluoride (PVDF) membrane

14 (Amersham Biosciences, Les Ulis, France). Membranes were then incubated with

15 antibodies directed to the phosphorylated forms of p38MAPK (Thr180/Tyr182), JNK

16 1/2 (Thr183/Tyr185), ERK 1/2(Thr202/Tyr204), NFk-B p65(Ser536) (all from Cell

17 Signaling Technology, Ozyme, St-Quentin-en-Yvelines, France) or to IкB- $\alpha$ (C-21,

18 Santa Cruz biotechnology, Santa Cruz, CA). Total p38MAPK was used as a loading

19 control with an antibody raised against total p38MAPK (Cell Signaling Technology).

20 After proper washes and incubation with secondary HRP-linked antibody,

21 immunoreactive bands were detected bychemiluminescence (ECL solution, Amersham

22 Biosciences) using a ChemiDoc XRS Imager (Bio-Rad, Marnes-La-Coquette, France).

23 Bands were quantified by densitometry using theImageLab software.

\section{$24 \quad$ T-lymphocyte proliferation}


1 The capacity of MoDCs to activate $\mathrm{T}$ cells has been evaluated by the use of T CD4+

2 T-cells lymphocytes labelled with $0.5 \mu \mathrm{M}$ CFSE following the manufacturer's

3 instructions. T cells are isolated from PBMC by positive selection with the MACS CD4

4 isolation kit (Miltenyi Biotec, Bergisch Gladbach, Germany). These $\mathrm{T}$ cells are

5 confirmed to have purity greater than 95\%, based on CD4 (555349, BD Biosciences)

6 expression evaluated by flow cytometry. MoDCs are pre-loaded with purified protein

7 derivative $(P P D)$ or not and then stimulated with NPs. Treated MoDCs were washed

8 and then cultured with autologous T cells at 3 different ratio a 1:8, 1:4, 1:2 DC/T-cell

9 ratio for 5 days in RPMI 1640 Glutamax supplemented with 10\% AB human serum, in

10 round bottomed 96-well plates. On day 5, CD4+T-cells proliferation is analyzed by

11 flow cytometry and expressed as the percentage of CFSE low $\mathrm{T}$ cells present in the 12 sample.

\section{Statistical analysis}

14 Significance of obtained results was calculated using nonparametric one-way analyses

15 of variance on ranks approach (Kruskall-Wallis) combined with Mann-Whitney U tests

16 for multiple comparisons (Statistica 7.0, Statsoft, Chicago, IL, USA). Data are

17 expressed as means \pm S.E.M. Effects were designated significant at $\mathrm{p}<0.05(*)$ or at $\mathrm{p}$ $18<0.001(* *)$.

\section{Results}

\section{PLGA nanoparticle properties}

21 As shown in Table 1, the selection of different stabilizers allowed the modification of

22 NP surface charge. PLGA/PF68 NPs had a mean diameter of $100 \mathrm{~nm}$, while both

23 PLGA/PVA and PLGA/CS NPs were around $200 \mathrm{~nm}$. All formulations had a narrow

24 size distribution, with a polydispersity index of 0.1-0.2. Transmission electron 
1 microscopic observations showed spherical NPs with a smooth surface. Zeta potential

2 measurements confirmed that the stabilizers influence NP surface charge. CS-coated

3 NPs exhibited a positive zeta potential $(+32 \pm 3 \mathrm{mV})$, whereas PVA-coated NPs were

4 almost neutral $(-5 \pm 1 \mathrm{mV})$, and PF68-coated NPs exhibited a negative zeta potential

$5(-24 \pm 1 \mathrm{mV})$. The presence of stabilizers on the NP surface was quantified by

6 spectrophotometric methods and ${ }^{1} \mathrm{H}$ NMR spectroscopy. Between $10 \mathrm{mg}$ and $30 \mathrm{mg}$ of

7 stabilizer (per $100 \mathrm{mg}$ of PLGA) was found to be associated with the NPs (Table 1).

8 The use of Rhod-PLGA to render fluorescent NPs did not influence their size

9 distribution nor their surface charge. The only exception was Rhod-PLGA/CS NPs, the

10 size and zeta potential of which slightly decreased (Table 1) due to the presence of

11 rhodamine moieties on the NP surface. An important point is to monitor the possible

12 aggregation of NPs in serum-containing media (Hinderliter et al., 2010), especially

13 since the PLGA NPs used in this study are expected to be transported by sedimentation

14 (DeLoid et al., 2017). The dispersion of the three NP types in fetal bovine serum-

15 containing cell culture medium resulted in only minor modifications of the mean

16 hydrodynamic diameters for up to $72 \mathrm{~h}$ incubation, and the same trends in zeta potential

17 values (Table S1), despite the complexity of the cell culture medium.

18 Cytotoxic effects of PLGA nanoparticles on dendritic cells

19 Our toxicological evaluation of PLGA NPs first consisted in the evaluation of the

20 cytotoxic impact of NP exposure on DCs. Results obtained by both the MTT test

21 (Figure 1) and the trypan blue dye exclusion assay (data not shown) showed that human

22 and murine DCs underwent about 5 to $10 \%$ cell death after $24 \mathrm{~h}$ of exposure to

23 concentrations as high as $0.5 \mathrm{mg} / \mathrm{ml}$ NPs. Given such relatively high NP concentrations

24 needed to induce only moderate cell death within the DC populations, these first results

25 clearly indicate that PLGA NPs exert very low cytotoxic effects on DCs. 


\section{$1 \quad$ PLGA nanoparticle uptake and internalization into dendritic cells}

2 NP uptake by DCs was assessed from a quantitative point of view using flow cytometry

3 by monitoring rhodamine-labelled NP fluorescence associated to DCs. In human DC

4 (MoDC), results show that PVA-coated PLGA NPs were the most efficiently taken up

5 by human DCs (Figure 2A). With these particles, fluorescence measured after $24 \mathrm{~h}$ of

6 exposure was about 70 times higher than cellular autofluorescence, while CS- and

7 PF68-coated PLGA NPs entry resulted in a 40-fold increase of cellular fluorescence.

8 Interestingly, fluorescence measurements carried out at early time points indicated that

9 for both PVA- and CS-coated NPs, the major part of the $24 \mathrm{~h} \mathrm{NP}$ entry within cells

10 occurred within the very first hours of exposure (about $75 \%$ after $3 \mathrm{~h}$ and $90 \%$ after

$116 \mathrm{~h}$ ). Conversely, PF68-coated NP uptake by cells went on according to a slower

12 mechanism since only $40 \%$ of the $24-\mathrm{h}$ NP entry within cells occurred within the first

136 hours of exposure. In murine DC (BMDC), Rresults show the major part of the $24 \mathrm{~h}$

14 NP entry within cells occurred after 3 hours of exposure. PVA-coated PLGA NPs were

15 also the most efficiently taken up by BMDC (Figure 2B). With these particles,

16 fluorescence measured after $24 \mathrm{~h}$ of exposure was about 50 times higher than cellular

17 autofluorescence compared to $40 \%$ with CS-coated NPs. Regarding PF68-coated NP,

18 uptake by BMDC is very low.

19 To better understand the PLGA NP interaction with DCs, we then used rhodamine-

20 labelled NPs to assess NP intracellular localization within DCs. Confocal microscopic

21 observations showed that, after $24 \mathrm{~h}$ of exposure, very large quantities of PLGA NPs

22 were encountered within the cytoplasm of both human DCs and murine (Figures 2A \& 23 B).

24 The herein demonstrated capacity of DCs to internalize large quantities of 25 PLGA NPs is in good accordance with their constitutive ability to constantly sample 
1 their surrounding environment since they act as sentinels of the immune system.

2 Interestingly, surfactants exert a clear effect on NP internalization, which might be due

3 to differences of the electric charge present at the surface of particles. According to our

4 present results, PVA-coated NPs, which are uncharged particles, are the most rapidly

5 and efficiently internalized. Regarding CS- and PF68-coated NPs, which are charged

6 particles, similar quantities of NPs entered the cells after a 24-h exposure period.

7 However, negatively charged particles (i.e. PF68-coated ones) seemed to be internalized

8 according to a slower kinetic pattern than both uncharged and positively charged

9 particles.

10 Surface protein modifications in dendritic cells after PLGA nanoparticle

11 exposure

12 To assess the phenotypic effects of NP exposure on DCs, we first studied the

13 modification of membrane markers of maturation. Obtained results clearly indicate that

14 a 24-h exposure to $200 \mu \mathrm{g} / \mathrm{mL}$ PLGA NPs induced moderate modifications of herein

15 tested maturation markers. Regarding human DCs (Figure 3A), we noticed subtle (fold

16 increases generally <2) but significant enhancement of the surface expression of the

17 CD83 maturation marker, the CD54 adhesion molecule (also known as Inter-Cellular

18 Adhesion Molecule 1, ICAM-1), as well as of costimulatory molecules such as the

19 CD40 receptor that interact with the CD40L of T cells and several members of the B7

20 family ligands (CD80/B7-1, CD86/B7-2 and PD-L1/CD274/B7-H1) that bind to the

21 CD28 family receptors of T cells. Similar results were obtained in murine DCs at the

22 surface of which expressions of CD40, CD86 and CCR7 (a chemokine receptor)

23 molecules were significantly increased (Figure 3B).

24 Both human and murine DCs can therefore be considered as in an activated

25 state, able to be engaged in immunological synapses with $\mathrm{T}$ cells. However, it is 
1 noticeable that PVA coated NPs generally exerted the most marked-slight effects effects

2 on murine DC phenotype compared to human DC. , while CS and PF68-coated NPs 3 induced only slight effects.

The effect of a soluble surfactant exposed to cells at an equivalent concentration to that associated to NPs was evaluated in the case of PVA vs. PVA-coated NPs (Figure

6 S1A). For all human DC markers, no significant increased was noticed for free PVA.

\section{$7 \quad$ Cytokine secretion profile of dendritic cells exposed to PLGA nanoparticles}

8 As a second approach of the phenotypic impact of PLGA NP exposure on DCs, we

9 evaluated the secretory activity of DCs (Figure 4). Eight cytokines and chemokines

10 were tested in the culture medium supernatants of human MoDCs collected after $24 \mathrm{~h}$ of 11 exposure to NPs. Results showed that the pro-inflammatory cytokines IL-1 $\beta$, IL-6, IL-8 and TNF- $\alpha$ were induced by human DCs. A similar induction was observed for the

13 CXCL10 and CCL5 chemokines (Figure 4A). Conversely, in murine BMDCs, no effect

14 on secretory activity of cells was observed as a result of NP exposure. Interleukine-6 15 and TNF- $\alpha$ cytokines even tended to be encountered in cell media at lower 16 concentrations when cells were exposed to PLGA NPs. However, such tendency was 17 not statistically significant (Figure 4B).

18 Our results therefore indicate that human MoDCs but not murine BMDCs 19 enhanced their cytokine secretory activity as a result of NP exposure. In human CDs, 20 the three categories of NPs induce the same cytokines (IL-6, IL-8 and CXCL10) in 21 identical proportions. In murine CDs, the base level of cytokine production is higher, therefore no effect is reported on cytokine production by the NPs. secretions since PF68-coated NPs generally exerted the slightest effects. As for maturation markers, the effects of PVA alone were tested (Figure S1B). With the 
1 exception of IL-8 secretion, no significant differences were observed compared to

2 control conditions, suggesting that surfactant effect was only due to its direct

3 association to NPs. In the case of IL-8, a slight but significant increase in its secretion

4 was noticed as compared to controls. However, effects of PVA alone were lower than

$5 \quad$ PVA-coated NP effects.

6 Investigation of signalling pathways activated as a result of PLGA nanoparticle

7 exposure

8 Considering the results obtained regarding both surface maturation marker expressions

9 and cytokine secretions, we then tried to elucidate the underlying signalling pathways

10 involved in DC maturation. We first carried out western blot experiments to follow the

11 time evolution of the intracellular contents of the phosphorylated forms of mitogen

12 activated protein kinases (p38MAPK, JNK 1/2, ERK 1/2) and of the p65 subunit of

$13 \mathrm{NF \kappa}-\mathrm{B}$, as well as the time evolution of I $\kappa \mathrm{B}-\alpha$ content, its cytoplasmic inhibitor (Figure

14 5). We showed that exposure to PVA- and PF68-coated PLGA NPs induce the

15 significant activation of both JNK and ERK 1/2 pathways as indicated by the increase

16 of the phosphorylated forms of these proteins in human DCs. Regarding CS-coated

17 PLGA NP exposure, JNK pathway was not activated but both p38 and ERK 1/2 kinases

18 were phosphorylated. NFK-B pathway study did not show any significant activation.

19 T lymphocyte proliferation is not modulated in response to PLGA nanoparticle

20 MoDCs pre-loaded purified protein derivative $(P P D)$ were treated with NPs, then

21 washed and cultured with purified autologous CD4+ T lymphocytes CD4+ T-cells cells

22 at 3 different ratio a 1:8, 1:4, 1:2 DC/T-cell ratio for 5 days. On day 5, CD4 cells CD4+

23 T lymphocytes-cells proliferation was analyzed by flow cytometry and expressed as the

24 percentage of CFSE low T cells. On day 5, <20\% of total cells were CFSElow at ratio 
$1 \quad 1 / 4$ in absence of NP. MoDC loaded with PPD and treated with LPS allow us to

2 appreciate the maximal of proliferation of the cells, $50 \%$ at ratio $1 / 4$. MoDCs preloaded

3 or not with PPD have a quit similar proliferation in regard to the ratio. NP did not affect

4 the proliferation of lymphocytes in response to MoDC preloaded with PPD whatever

5 the ratio tested (Figure 6). These results suggest that NP-stimulated MoDCs do not

6 augment the proliferation of $\mathrm{CD}^{+} \mathrm{T}$-cells lymphocytes. Collectively, these findings

7 reveal that PLGA NPs do not induce the capacity of mature moDC to activate

8 autologous CD4+ T cells proliferation.

\section{Discussion}

10 The use of NPs in medicine requires the understanding of their possible adjuvant effect

11 in the immune response. In the particular case of PLGA materials, some questions

12 regarding immune consequences of their use as NP carriers still remain unclear. It is

13 currently accepted that charging professional APC with antigens loaded onto PLGA

14 vehicles leads to a presentation of MHC class I-antigen complexes over an extended

15 period (Audran et al., 2003). More generally, PLGA NP carriers are considered as

16 efficient carriers of antigens in comparison with the use of soluble forms of antigens

17 (Diwan et al., 2003). However, to this day, professional APC activation has not been

18 fully investigated in response to PLGA NP. The current study was therefore conducted

19 to examine PLGA NP effects on DCs. A particular emphasis was placed on the impact

20 of polyelectrolyte coatings on the particle uptake by cells, as well on the phenotype and

21 secretory profile of DCs. To this purpose, PLGA NPs were coated either with CS, PVA, or PF68 stabilizers, resulting in significant differences in their zeta potential (the resulting particles being either cationic, neutral or anionic, respectively). charge, all herein tested NP formulations were efficiently taken up and internalized by 
1 DCs, as evidenced by cytometry analysis and confocal microscopy observations,

2 respectively. Uptake of PLGA nano- and micro-particles has previously been

3 demonstrated in vitro by human peripheral blood-monocytes-derived DCs (Walter et al.,

4 2001; Lustiak et al., 2002; Waeckerle-Men et al., 2004; Yoshida \& Babensee, 2006b;

5 Fischer et al., 2007; Ma et al., 2011), human cord-blood CD34+ stem cells-derived DCs

6 (Diwan et al., 2003), mouse bone marrow-derived DCs (Elamanchili et al., 2004; Ma et

7 al., 2011), and in vivo upon intradermal immunization (Newman et al., 2002). Some of

8 these authors additionally reported that PLGA NPs were not detectable inside the DCs

9 when cells were pretreated with cytochalasin, an inhibitor of actin polymerization, or

10 when incubation with the fluorospheres was carried out at $4^{\circ} \mathrm{C}$, therefore demonstrating

11 that NP internalization is achieved by a phagocytosis phenomenon (Lustiak et al., 2002;

12 Yoshida \& Babensee, 2006b). This conclusion was supported by electron microscopy

13 studies, where membrane ruffling around the phagocytosed particle was visualized

14 (Diwan et al., 2003). According to Hamdy et al. (2011), the uptake of PLGA particles is

15 affected by surface charge. Cationic particles would indeed be particularly effective for

16 uptake by DCs. The ionic attraction between the positively charged particles and the

17 negatively charged cell surface initiates efficient binding and facilitate particle

18 internalization, even though the ossible formation of a protein corona around the NPs in

19 serum-containing media should be taken into account (Tenzer et al., 2011). In our study,

20 cationic (CS-coated) NPs remained positively-charged in cell culture media, and were

21 indeed rapidly engulfed by DCs since about $80 \%$ of the particles that were encountered

22 into the cells after $24 \mathrm{~h}$ of exposure actually entered the cells within the first $3 \mathrm{~h}$ of

23 incubation. Anionic (PF68-coated) NPs were taken up by DCs according to a slower

24 kinetics. Despite this difference, after $24 \mathrm{~h}$ of incubation, both anionic and cationic

25 particles were internalized to a similar extent on a quantitative basis. Neutral (PVA- 
1 coated) NPs appeared as the most efficiently internalized particles since DC

2 fluorescence was about twice as high compared to CS- or PF68-coated NP exposed cells

3 after $24 \mathrm{~h}$. Such a difference shows that electrostatic interactions cannot account alone

4 for internalization by cells, and could be related to the fact that neutral particles are less

5 susceptive to attract surrounding molecules such as proteins that are present in the

6 culture medium.

7 As for their cytotoxic potential, PLGA NPs do not significantly affect the 8 survival of DCs whatever their charge, even when the cells were loaded with very large

9 amounts of NPs, which is in total agreement with others studies (Walter et al., 2001;

10 Waeckerle-Men et al., 2004; Fischer et al., 2007; Semete et al., 2010). Previous studies

11 on PLGA NPs displaying positive, negative or neutral surface charge revealed that

12 positively-charged NPs appeared to be more cytotoxic and genotoxic to different cell

13 lines with ROS induction linked to high endocytosis and chromosomal aberrations,

14 contrary to other NP types that appear to be relatively inert (Platel et al., 2016).

15 Noticeable phenotypic changes induced in both human and murine DCs in 16 response to PLGA NP exposure as evidenced by significant upregulations (CD86, 17 CD80, CD40, CD83), are consistent with a change allowing the transition from an 18 antigen-capturing to an antigen-presenting state of DCs (Banchereau and Steinman, 19 1998). The observed effects are attributed to PLGA NP free of endotoxin since the 20 endotoxin levels were below the USP prescribed content in water for injection $(0.25$ 21 endotoxin units/mL, as tested by the LAL assay). Even if all tested PLGA NPs had an 22 impact on DC maturation, PVA NPs were the most potent since they exerted a higher 23 induction of all surface markers (i.e. CD40, CD54, CD80, CD83, CD86 and PDL-1 in 24 MoDCs; CD40, CD86 and CCR7 in BMDCs). This maturation can be correlated with 25 the higher and rapid phagocytosis of PVA-coated NPs. However, Human DCs are more 
1 sensitive than murine DCs in response to nanoparticles since many surface markers are

2 induced, as are cytokines.

Although some literature describes the absence of DC maturation upon PLGA

4 particle exposure, both in MoDCs (Walter et al., 2001; Waeckerle-Men et al., 2004;

5 Fischer et al., 2007; Bivas-Benita et al., 2009) and BMDCs (Sun et al., 2003; Clawson

6 et al., 2010; Ma et al., 2011), our study confirms a large number of studies

7 demonstrating phenotypical changes induced in both human and murine DCs exposed to

8 PLGA films and particles (Yoshida \& Babensee, 2004, 2006a; Babensee \& Paranjpe,

9 2005; Diwan et al., 2003; Sun et al., 2003; Elmanchili et al., 2004; Yoshida et al., 2007).

10 Moreover, we demonstrate a relationship between PLGA particle charge and the

11 acquired DC phenotype, which remained an open question in several studies (Fischer et

12 al., 2007; Jilek et al., 2004).

13 Beside the expression of maturation markers, the secretion of cytokines is

14 indeed crucial for T-cell activation and efficient antigen presentation. We therefore

15 investigated the influence NPs on cytokine secretion of DCs. Our results clearly

16 demonstrated that, upon exposure to PLGA NPs, MoDCs secrete significant amounts of

17 pro-inflammatory cytokines (IL-1 $\beta$, IL-6, IL-8 and TNF- $\alpha$ ) and chemokines (CXCL10,

18 CCL5), consistent with the maturation of human DCs upon exposure to PLGA NPs, and

19 with other studies demonstrating the impact of PLGA particles on cytokine secretion by

20 DCs. The induction of IL-6, IL-8 and TNF- $\alpha$ by PLGA-exposed MoDCs may control a

21 TH1 response as suggested elsewhere (Yoshida \& Babensee, 2006a,b). This result is in

22 agreement with another study in which the delivery of a model antigen with PLGA

23 particles resulted in a TH1 response as measured by production of $\operatorname{IgG} 2$ isotype

24 antibody against the antigen (Newman et al., 1998). 
2 MoDC) equally. The differences mainly observed in cytokine production by MoDCs

3 might be explained by the origin of DC. Human MoDCs are inflammatory monocyte-

4 derived DC close to conventional DC while murine DCs are differentiated from CD34.

The effect of the surfactant themselves have been raised in previous reports on

6 PLGA NPs (Guedj et al., 2015; Menon et al., 2012). Our results show that the effects of

7 PVA alone, exposed at an equivalent concentration to that associated to NPs, had no

8 own effect on DC maturation. This can be related to previous observations that PVA

9 remains strongly associated to PLGA NPs even after thorough purification (Spek et al.,

10 2015). Regarding the contribution of the PLGA core on the cell response, previous

11 reports by our group (Grabowski et al., 2015) showed that dendritic cell surrogates do

12 not show any difference in terms of mitochondrial activity and necrosis induction

13 following exposure of PVA-coated PLGA nanoparticles and surfactant-free PLGA

14 nanoparticles, which were obtained by a specific preparation technique, at concentration

15 ranges used in the present study $(<1 \mathrm{mg} / \mathrm{mL})$.

16 Phenotypic changes and concomitant cytokine secretion are maturation

17 hallmarks that result from intracellular signaling cascades. Different signaling pathways

18 such as MAPKs and NF-kB play important roles in DC maturation (Neves et al., 2011).

19 Regarding the maturation process, it has been proved that different chemicals could

20 stimulated different signal transduction pathways and subsequently induced different

21 phenotypic and functional changes in DC, such as MAPKs and NF- $\kappa \mathrm{B}$ signalling

22 pathways (Antonios et al., 2010). In our hands, MAPK pathways were activated in

23 response to all PLGA NPs, but at different levels depending on the type of PLGA NPs.

24 Our results show a nice correlation between MAPKs and human DC maturation induced

25 by PLGA NPs. 
1 In addition, we show that PLGA NP do not affect the activation of $\mathrm{T}$ lymphocytes in

2 response to an antigen such as PPD. This confirms these NP do not modify the adaptive

3 immune response of DC even if the phenotype is slightly activated.

\section{Conclusions}

6 Our study indicates that PLGA-NPs induce clear consequences on the pivotal 7 properties of DCs in vitro. Exposure of immature DCs to three PLGA NP types with

8 different surface coatings and surface charge resulted in marked particle uptake by the

9 cells, but no significant cell death. Nevertheless, these NPs were not as inert as expected

10 since alterations in DC phenotype were reported, as assessed by the expression levels of

11 cell surface markers of DC maturation. In addition, the functional secretory activity of

12 DCs was largely altered upon phagocytosis of the different NPs. The study presented

13 herein lays a foundation for studying the effects of PLGA nanomaterials on the immune

14 system prior to their use as vehicles in nanomedicine. This study allowed us to indicate

15 that interspecific differences are important to take into account since human activation

16 of CDs is higher than that of murine CDs. Human DCs are and This is the first study

17 that demonstrates MAPK activation by PLGA exposure in DCs, and the potential role

18 of surfactants in the effectively taken pathways. Further studies are needed to study the

19 role of encapsulated bioactive compounds on the effects of such PLGA nanocarriers,

20 which are relevant for drug and vaccine delivery purposes.

\section{Acknowledgements}

23 This study was supported by the the ANSM "Emerging risks" program and the Agence

24 Nationale de la Recherche (under reference Nanobiotox). 


\section{Declaration of interest}

2 The authors report no conflicts of interest. The authors alone are responsible for the

3 content and writing of the paper.

\section{References}

5 AFSSAPS (Agence Française de Sécurité SAnitaire des Produits de Santé). 2009. Recommendations for toxicological evaluation of nanoparticle medicinal products, p. 8 .

Antonios, D., Rousseau, P.; Larange, A, Kerdine-Romer, S., Pallardy, M. 2010. Mechanisms of IL-12 Synthesis by Human Dendritic Cells Treated with the Chemical Sensitizer NiSO4. J Immunol 185(1):89-98.

Aragao-Santiago, L., Hillaireau, H., Grabowski, N., Mura, S., Nascimento, T. L., Dufort, S., Coll, J.-L., Tsapis, N., Fattal, E. 2015. Compared in vivo toxicity in mice of lung delivered biodegradable and non-biodegradable nanoparticles. Nanotoxicology 10(3):292-302.

Audran, R., Peter, K., Dannull, J., Men, Y., Scandella, E., Groettrup, M., Gander, B., and Corradin, G. 2003. Encapsulation of peptides in biodegradable microspheres prolongs their MHC class-I presentation by dendritic cells and macrophages in

Babensee, J. E., Paranjpe, A. 2005. Differential levels of dendritic cell maturation on different biomaterials used in combination products. J Biomed Mater Res A 74:503-10.

Bala, I., Hariharan, S., Kumar, M. N. 2004. PLGA nanoparticles in drug delivery: the state of the art. Crit Rev Ther Drug Carrier Syst 21:387-422.

Banchereau, J., Steinman, R. M. 1998. Dendritic cells and the control of immunity. Nature 392:245-52. 
1 Bhardwaj, V., Hariharan, S., Bala, I., Lamprecht, A., Kumar, N., Panchagnula, R., 2 Kumar, M. N. 2005. Pharmaceutical aspects of polymeric nanoparticles for oral delivery. Journal of Biomedical Nanotechnology 1:235-258.

Bivas-Benita, M., Lin, M. Y., Bal, S. M., van Meijgaarden, K. E., Franken, K. L. M. C., Friggen, A. H., Junginger, H. E., Borchard, G., Klein, M. R., Ottenhoff, T. H. M. 2009. Pulmonary delivery of DNA encoding Mycobacterium tuberculosis latency antigen Rv1733c associated to PLGA-PEI nanoparticles enhances $\mathrm{T}$ cell responses in a DNA prime/protein boost vaccination regimen in mice. Vaccine 27:4010-4017.

Blank, F., Stumbles, P., von Garnier, C. 2011. Opportunities and challenges of the pulmonary route for vaccination. Expert Opin Drug Deliv 8:547-63.

Bisht R, Rupenthal ID. 2016. PLGA nanoparticles for intravitreal peptide delivery: statistical optimization, characterization and toxicity evaluation. Pharm Dev Technol. 3:1-10

Clawson, C., Huang, C. T., Futalan, D., Martin Seible, D., Saenz, R., Larsson, M., Ma, W., Minev, B., Zhang, F., Ozkan, M., Ozkan, C., Esener, S., and Messmer, D. 2010. Delivery of a peptide via poly(d,1-lactic-co-glycolic) acid nanoparticles enhances its dendritic cell-stimulatory capacity. Nanomedicine 6(5):651-661.

Chen, X. and Changyou, G. 2018. Influences of surface coating of PLGA nanoparticles on immune activation of macrophages J. Mater. Chem. B, 6, 2065-2077

Dailey, L. A., Jekel, N., Fink, L., Gessler, T., Schmehl, T., Wittmar, M., Kissel, T., Seeger, W. 2006. Investigation of the proinflammatory potential of biodegradable nanoparticle drug delivery systems in the lung. Toxicol Appl Pharmacol 215:1008. 
1 DeLoid, G.M., Cohen, J.M., Pyrgiotakis, G., Demokritou, P. 2017. Preparation, 2 characterization, and in vitro dosimetry of dispersed, engineered nanomaterials. Nat Protoc, 12(2):355-371

4 Di Gioacchino, M., Petrarca, C., Lazzarin, F., Di Giampaolo, L., Sabbioni, E., Boscolo, P., Mariani-Costantini, R., Bernardini, G. 2011. Immunotoxicity of nanoparticles. Int J Immunopathol Pharmacol 24:65S-71S.

7 Diwan, M., Elamanchili, P., Lane, H., Gainer, A., Samuel, J. 2003. Biodegradable nanoparticle mediated antigen delivery to human cord blood derived dendritic cells for induction of primary T cell responses. J Drug Target 11:495-507.

10 Dobrovolskaia, M. A., Germolec, D. R., Weaver, J. L. 2009. Evaluation of nanoparticle 11 immunotoxicity. Nat Nanotechnol 4:411-4.

12 Dobrovolskaia, M. A., McNeil, S. E. 2007. Immunological properties of engineered nanomaterials. Nat Nanotechnol 2:469-78.

14 Dobrovolskaia, MA., Shurin, M., Shvedova, A. 2016. Current understanding of interactions between nanoparticles and the immune system. Toxicol Appl Pharm 299:78-89

El Ali, Z., Delomenie, C., Botton, J., Pallardy, M., Kerdine-Romer, S. 2017. Dendritic cells' death induced by contact sensitizers is controlled by Nrf2 and depends on glutathione levels. Toxicol Appl Pharmacol 322:41-50

Elamanchili, P., Diwan, M., Cao, M., Samuel, J. 2004. Characterization of poly(,-lacticco-glycolic acid) based nanoparticulate system for enhanced delivery of antigens to dendritic cells. Vaccine 22:2406-2412.

Fischer, S., Uetz-von Allmen, E., Waeckerle-Men, Y., Groettrup, M., Merkle, H. P., Gander, B. 2007. The preservation of phenotype and functionality of dendritic 
cells upon phagocytosis of polyelectrolyte-coated PLGA microparticles. Biomaterials 28:994-1004.

3 Gross, O., Thomas, C. J., Guarda, G., Tschopp, J. 2011. The inflammasome: an integrated view. Immunol Rev 243:136-51.

5 Grabowski, N., Hillaireau, H., Vergnaud, J., Tsapis, N., Pallardy, M., Kerdine-Römer, S., Fattal, E. 2015. Surface coating mediates the toxicity of polymeric nanoparticles towards human-like macrophages. Int J Pharm. 30;482(1-2):75-83

Guedj, E. et al. 2015. Preparation, characterization, and safety evaluation of poly(lactide-co-glycolide nanoparticles for protein delivery into macrophages. Int J Nanomedicine 10:5965-79

Hamdy, S., Haddadi, A., Hung, R. W., Lavasanifar, A. 2011. Targeting dendritic cells with nano-particulate PLGA cancer vaccine formulations. Adv Drug Deliv Rev 63:943-55.

Hinderliter, P.M., Minard, K.R., Orr, G., Chrisler, W.B., Thrall, B.D., Pounds, J.G., Teeguarden, J.G. 2010. ISDD: A computational model of particle sedimentation, diffusion and target cell dosimetry for in vitro toxicity studies. Part Fibre Toxicol. 7: 36.

Ilinskaya AN(1), Dobrovolskaia MA. 2014. Immunosuppressive and anti-inflammatory properties of engineered nanomaterials. Br J Pharmacol. 171(17):3988-4000.

Jilek, S., Ulrich, M., Merkle, H. P., Walter, E. 2004. Composition and surface charge of DNA-loaded microparticles determine maturation and cytokine secretion in human dendritic cells. Pharm Res 21:1240-7.

Larange, A.; Antonios, D.; Pallardy, M.; Kerdine-Romer, S. 2012. Glucocorticoids inhibit dendritic cell maturation induced by Toll-like receptor 7 and Toll-like receptor 8. J Leukoc Biol 91(1):105-117. 
1 Lu, J. M., Wang, X., Marin-Muller, C., Wang, H., Lin, P. H., Yao, Q., Chen, C. 2009.

2 Current advances in research and clinical applications of PLGA-based 3 nanotechnology. Expert Rev Mol Diagn 9:325-41.

4 Lutsiak, M. E., Robinson, D. R., Coester, C., Kwon, G. S., Samuel, J. 2002. Analysis of poly(D,L-lactic-co-glycolic acid) nanosphere uptake by human dendritic cells and macrophages in vitro. Pharm Res 19:1480-7.

Ma, W., Smith, T., Bogin, V., Zhang, Y., Ozkan, C., Ozkan, M., Hayden, M., Schroter,

Menon et al.. 2012 Effects of surfactants on the properties of PLGA nanoparticles J Biomed Mater Res A. 100:1998-2005

Mura, S., Hillaireau, H., Nicolas, J., Kerdine-Romer, S., Le Droumaguet, B.,

Neves, B. M., Gonçalo, M., Figueiredo, A., Duarte, C. B., Lopes, M. C., Cruz, M. T. 2011. Signal transduction profile of chemical sensitisers in dendritic cells: An 
endpoint to be included in a cell-based in vitro alternative approach to hazard identification? Toxicology and Applied Pharmacology 250:87-95.

3 Newman, K. D., Elamanchili, P., Kwon, G. S., Samuel, J. 2002. Uptake of poly(D,Llactic-co-glycolic acid) microspheres by antigen-presenting cells in vivo. J Biomed Mater Res 60:480-6.

6 Newman, K. D., Samuel, J., Kwon, G. 1998. Ovalbumin peptide encapsulated in poly(d,1 lactic-co-glycolic acid) microspheres is capable of inducing a $\mathrm{T}$ helper type 1 immune response. J Control Release 54:49-59.

9 Oostingh, G. J., Casals, E., Italiani, P., Colognato, R., Stritzinger, R., Ponti, J., Pfaller,

Petrarca, C. et al, 2015 Engineered metal based nanoparticles and innate immunity. Clin

$$
\text { Mol Allergy 15;13:13 }
$$

Platel et al.. 2016. Influence of the surface charge of PLGA nanoparticles on their in vitro genotoxicity, cytotoxicity, ROS production and endocytosis, J. Appl. Toxicol. 36: 434-444

Semete, B., Booysen, L., Lemmer, Y., Kalombo, L., Katata, L., Verschoor, J., Swai, H. S. 2010. In vivo evaluation of the biodistribution and safety of PLGA nanoparticles as drug delivery systems. Nanomedicine: NBM 6:662-671. 
1 Spek et al. 2015. Characterisation of PEGylated PLGA nanoparticles comparing the nanoparticle bulk to the particle surface using UV/Vis spectroscopy, SEC, $1 \mathrm{H}$ NMR spectroscopy, and X-ray photoelectron spectroscopy. Applied Surface Science 347:378-385

Sun, H., Pollock, K. G. J., Brewer, J. M. 2003. Analysis of the role of vaccine adjuvants in modulating dendritic cell activation and antigen presentation in vitro. Vaccine 21:849-855.

Tenzer, S., Docter, D., Rosfa, S., Wlodarski, A., Kuharev, J., Rekik, A., Knauer, S.K., Bantz, C., Nawroth, T., Bier, C., Sirirattanapan, J., Mann, w., Treuel, L., Zellner, R., Maskos,M., Schild, H., Stauber R.H.. 2011. Nanoparticle Size Is a Critical Physicochemical Determinant of the Human Blood Plasma Corona: A Comprehensive Quantitative Proteomic Analysis. ACS Nano 5 (9), 7155-7167

Waeckerle-Men, Y., Scandella, E., Uetz-von Allmen, E., Ludewig, B., Gillessen, S., Merkle, H. P., Gander, B., Groettrup, M. 2004. Phenotype and functional analysis of human monocyte-derived dendritic cells loaded with biodegradable poly(lactide-co-glycolide) microspheres for immunotherapy. J Immunol Methods 287:109-124.

Walter, E., Dreher, D., Kok, M., Thiele, L., Kiama, S. G., Gehr, P., Merkle, H. P. 2001. Hydrophilic poly(-lactide-co-glycolide) microspheres for the delivery of DNA to human-derived macrophages and dendritic cells. J Control Release 76:149-168.

Xu, P., Gullotti, E., Tong, L., Highley, C. B., Errabelli, D. R., Hasan, T., Cheng, J. X., Kohane, D. S., Yeo, Y. 2009. Intracellular drug delivery by poly(lactic-coglycolic acid) nanoparticles, revisited. Mol Pharm 6:190-201.

Yoshida, M., Babensee, J. E. 2004. Poly(lactic-co-glycolic acid) enhances maturation of human monocyte-derived dendritic cells. J Biomed Mater Res A 71:45-54. 
1 Yoshida, M., Babensee, J. E. 2006a. Differential effects of agarose and poly(lactic-co2 glycolic acid) on dendritic cell maturation. J Biomed Mater Res A 79:393-408.

3 Yoshida, M., Babensee, J. E. 2006b. Molecular aspects of microparticle phagocytosis $4 \quad$ by dendritic cells. J Biomater Sci Polym Ed 17:893-907.

5 Yoshida, M., Mata, J., Babensee, J. E. 2007. Effect of poly(lactic-co-glycolic acid) 6 contact on maturation of murine bone marrow-derived dendritic cells. J Biomed $7 \quad$ Mater Res A 80:7-12.

8 Yue, Z.G. et al. 2011. Surface Charge Affects Cellular Uptake and Intracellular 9 Trafficking of Chitosan-Based Nanoparticles. Biomacromolecules 12:2440-2446

10 Zolnik, B. S., Gonzalez-Fernandez, A., Sadrieh, N., Dobrovolskaia, M. A. 2010. 11 Minireview: Nanoparticles and the Immune System. Endocrinology 151:458-465. 


\section{Table}

2 Table 1. PLGA nanoparticle characterization.

3 Data are presented as mean \pm SD. Abbreviations: CS, chitosan; PF68, poloxamer 188;

4 PLGA, poly(lactide-co-glycolide); PVA, poly(vinyl alcohol); rhod, rhodamine B; TEM,

5 transmission electron microscopy.

6 
$1 \quad$ Figures captions

2 Figure 1. Effects of PLGA NPs on cell viability. Human MoDCs (solid lines) and murine BMDCs (dashed lines) were exposed to PLGA NPs $(0-500 \mu \mathrm{g} / \mathrm{mL})$ for $24 \mathrm{~h}$. Cell death was assessed by the MTT test.

Figure 2. PLGA nanoparticle uptake and internalization. A. Confocal microscopy observations. Up. Human DCs were exposed for $24 \mathrm{~h}$ to rhodamine coated-PLGA NPs (200 $\mu \mathrm{g} / \mathrm{mL})$. From left to right: PLGA/PVA, PLGA/CS, PLGA/PF68. Lysosomes were labeled using a green LysoTracker® probe (InVitroGen). Flow cytometry fluorescence analysis Down. Human DCs were exposed for 3, 6 or $24 \mathrm{~h}$ to rhodamine-coated PLGA NPs $(0-400 \mu \mathrm{g} / \mathrm{mL})$. From left to right: PLGA/PVA, PLGA/CS, PLGA/PF68. Red fluorescence was then measured by flow cytometry. Fluorescence intensity of NP exposed cells was then divided by fluorescence intensity of control cells (i.e. autofluorescence). B. Confocal microscopy observations. Up. Murine DCs were exposed for $24 \mathrm{~h}$ to rhodamine coated-PLGA NPs $(200 \mu \mathrm{g} / \mathrm{mL})$. From left to right: PLGA/PVA, PLGA/CS, PLGA/PF68. Lysosomes were labeled using a green LysoTracker ${ }^{\circledR}$ probe (InVitroGen). Flow cytometry fluorescence analysis Down. Murine DCs were exposed for 3, 6 or $24 \mathrm{~h}$ to rhodamine-coated PLGA NPs (0-400 $\mu \mathrm{g} / \mathrm{mL})$. From left to right: PLGA/PVA, PLGA/CS, PLGA/PF68. Red fluorescence was then measured by flow cytometry. Fluorescence intensity of NP exposed cells was then divided by fluorescence intensity of control cells (i.e. autofluorescence).

Figure 3. Phenotypic analysis of dendritic cells treated by PLGA NPs. Human MoDCs (A) and murine BMDCs (B) were exposed for $24 \mathrm{~h}$ to distilled water (unexposed cells, white), PLGA NPs $(200 \mu \mathrm{g} / \mathrm{mL}$, grey), or lipopolysaccharide (LPS, $25 \mathrm{ng} / \mathrm{mL}$, positive control, black). Phenotypic markers of maturation were then studied using flow cytometry analysis of fluorescent immunolabelling (statistically significant differences from unexposed cells: grey star, $\mathrm{p}<0.1$; black star, ${ }^{*}, \mathrm{p}<0.05 ; * * ; \mathrm{p}<0.01$, $n=5$ ).

Figure 4. Cytokine production by DCs. MoDCs (A) and murine BMDCs (B) were exposed for $24 \mathrm{~h}$ to distilled water (unexposed cells, white), PLGA NPs (200 $\mu \mathrm{g} / \mathrm{mL}$, grey), or lipopolysaccharide (LPS, $25 \mathrm{ng} / \mathrm{mL}$, positive control, black). Cytokine concentrations were then measured in exposure medium using the cytometric bead array 
1 technique (statistically significant differences from unexposed cells: grey star, $\mathrm{p}<0.1$;

2 black star, $\mathrm{p}<0.05$; two stars, $\mathrm{p}<0.001 ; \mathrm{n}=5$ ).

3 Figure 5. PLGA NPs modulate MAPK phosphorylation in MoDCs. Human MoDCs 4 were exposed for $15 \mathrm{~min}, 30 \mathrm{~min}, 1 \mathrm{~h}$ or $2 \mathrm{~h}$ to distilled water (unexposed cells, white ), 5 PLGA NPs (grey ,200 $\mu \mathrm{g} / \mathrm{mL}$ ), or lipopolyssacharide (LPS, $25 \mathrm{ng} / \mathrm{mL}$, positive 6 control, black). Cells were lysed and the level of phosphorylated p38MAPK, JNK and 7 ERK was evaluated by western-blotting. Membrane was then probed with anti8 p38MAPK Ab for loading control. A. Results of a representative experiment B. 9 Quantitative time evolution of phosphorylated MAPKs. Folds represent the ratio of 10 normalized intensity of specific band divided by the normalized intensity of unexposed 11 cells (statistically significant differences from unexposed cells: grey star, $\mathrm{p}<0.1$; black 12 star, $\mathrm{p}<0.05 ; *, \mathrm{p}<0.05 ; * * ; \mathrm{p}<0.01 \mathrm{n}=5)$.

14 Figure 6. T lymphocyte proliferation is not modulated in response to PLGA NPs.

15 MoDCs pre-loaded with purified protein derivative $(P P D)$ or not were stimulated with 16 NPs. Treated MoDCs were washed and then cultured with CFSE-loaded CD4 ${ }^{+} \mathrm{T}$ 17 autologous $\mathrm{T}$ cells at 3 different ratio a 1:8, 1:4, 1:2 DC/T-cell ratio for 5 days. On day 18 5, CD4+ T-cells proliferation was analyzed by flow cytometry and expressed as the 19 percentage of CFSE low T cells present in the sample $(n=2)$. 